\title{
MEASURING HEALTH INEQUALITIES IN NEW SOUTH WALES
}

Helen Moore and Louisa Jorm

Epidemiology and Surveillance Branch

NSW Department of Health

This paper presents information on some key indicators of inequality in health in NSW related to demographic, socioeconomic and geographic factors. Its purposes are to highlight some of the more striking health inequalities, and to describe some of the challenges in improving their measurement.

The information presented here is drawn from the reports The health of the people of New South Wales-Report of the Chief Health Officer 2000, ${ }^{1}$ and the electronic report NSW Health Surveys 1997 and 1998. ${ }^{2}$ More detailed information about a wide range of health inequalities is available in these reports.

\section{HEALTH INEQUALITIES BY SEX}

Measurement of health inequalities between males and females is relatively simple because sex is available in all the major health data sources in NSW. These demonstrate substantial differences in health, and use of health services, between males and females. For example:

- Women have a longer life expectancy than men, although this difference is decreasing. Between 1965 and 1998, life expectancy at birth steadily increased from 67.1 to 76.5 years for males, and from 73.7 to 81.9 years for females.
- In the 1997 and 1998 NSW Health Surveys, women were more likely to report being admitted to hospital overnight and to report visiting a general practitioner in the last two weeks and the last 12 months, whereas men were more likely to report visiting an emergency department in the last 12 months.

- In the same surveys, men were more likely than women to report being current smokers and being overweight or obese. Men were less likely to report eating the recommended daily quantities of vegetables and fruit. However, fewer women than men reported adequate levels of physical activity.

\section{HEALTH INEQUALITIES BY COUNTRY OF BIRTH AND LANGUAGE SPOKEN AT HOME}

Measuring health inequalities among country-of-birth and language groups is not straightforward in NSW. Data on language spoken at home is not available in some data sets (for example, Australian Bureau of Statistics mortality data), and the accuracy of ethnicity data in others (such as the NSW Inpatients Statistics Collection) is unknown. Other limitations include the restricted availability of population denominator data (available only every five years from the Census) for calculation of rates, and the small size of many ethnic communities.

Available data demonstrate that in general, overseasborn residents have better health than Australian-born

\section{FIGURE 1}

PREMATURE BIRTHS BY COUNTRY OF BIRTH OF MOTHER

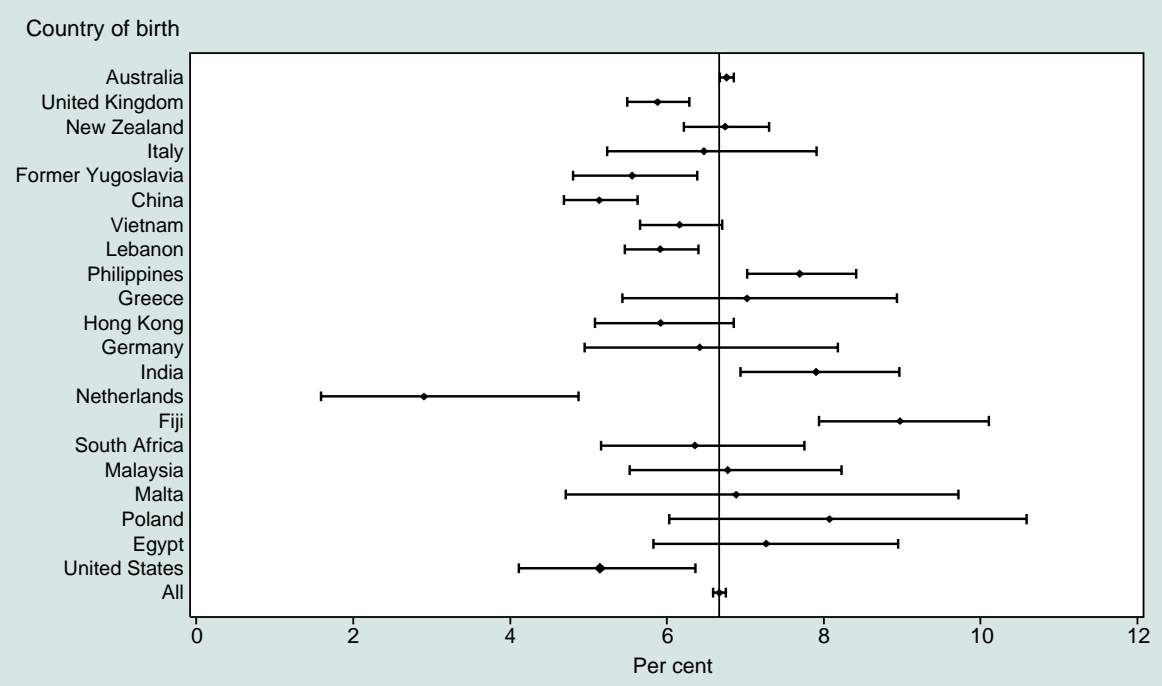

Note: Births where gestational age was less than 37 weeks were classified as premature births. Infants of at least 400 grams birth weight or at least 20 weeks gestation were included. Upper and lower limits of the 95 per cent confidence interval for the point estimate least 20 weeks gestation were included. Upper and lower limits of the 95 per cent confidence interval for the point estimate

Source: NSW Midwives Data Collection (HOIST). Epidemiology and Surveillance Branch, NSW Department of Health. 
residents, possibly reflecting a 'healthy migrant effect' ${ }^{3}$ Rates of premature death, chronic diseases and recent illnesses tend to be lower for migrants. However, certain diseases and risk factors are more prevalent among some country-of-birth groups. Some key examples are:

- In the period 1994 to 1998 , premature births varied by maternal country of birth, from 3.3 per cent for mothers born in the Netherlands to 8.8 per cent for mothers born in Fiji. Mothers born in the United Kingdom and Ireland, countries of the former Yugoslavia and China were less likely to give birth prematurely, while mothers born in Lebanon and Malta were more likely to have premature births (Figure 1).

- In 1997 and 1998, men and women born in New Zealand and men born in Vietnam and Lebanon, reported higher rates of current smoking than their Australian-born counterparts. Men and women born in Italy and women born in China, Vietnam and the Philippines, were less likely to report current smoking.

- While cervical cancer rates were higher in women born in China and Vietnam in 1993-1997 compared with Australian-born women, self-reported Pap Test screening rates were lower, particularly for women born in China.

- There were considerable differences in reported rates of toothache (sometimes, often or very often) in the past 12 months among language groups. Men and women speaking Chinese and Lebanese, and men speaking Vietnamese, reported higher than average rates of toothache (Figure 2).

\section{HEALTH INEQUALITIES BY INDIGENOUS STATUS}

Indigenous status is generally poorly recorded in most health data collections; however, improvements have been made in recent times, particularly for death data. Additionally, examination of trends in indigenous health is complicated by increasing levels of self-identification as an indigenous person. This affects both health datasets and population denominator data. ${ }^{4}$ Despite these limitations, poorer birth and health outcomes and higher prevalence of health risk factors among indigenous people have long been recorded and remain apparent in NSW. Some of the more striking differences include:

- There is currently little information about the mental health and wellbeing of indigenous Australians, nor is there an agreed method for assessing it. ${ }^{4}$ However, in the 1997 and 1998 NSW Health Surveys, ${ }^{2}$ the reported level of psychological distress, based on the Kessler 10 measure, ${ }^{5}$ was higher among indigenous than non-indigenous respondents of both sexes (Figure 3).

- Among people who reported having an overnight hospital admission in the last 12 months, indigenous people (19.7 per cent) were more than twice as likely

\section{FIGURE 2}

\section{TOOTHACHE EXPERIENCE BY LANGUAGE SPOKEN AT HOME}

Toothache experienced very often, often and sometimes in previous 12 months by language spoken at home and sex, persons aged 16 years and over with at least one natural tooth, NSW 1998
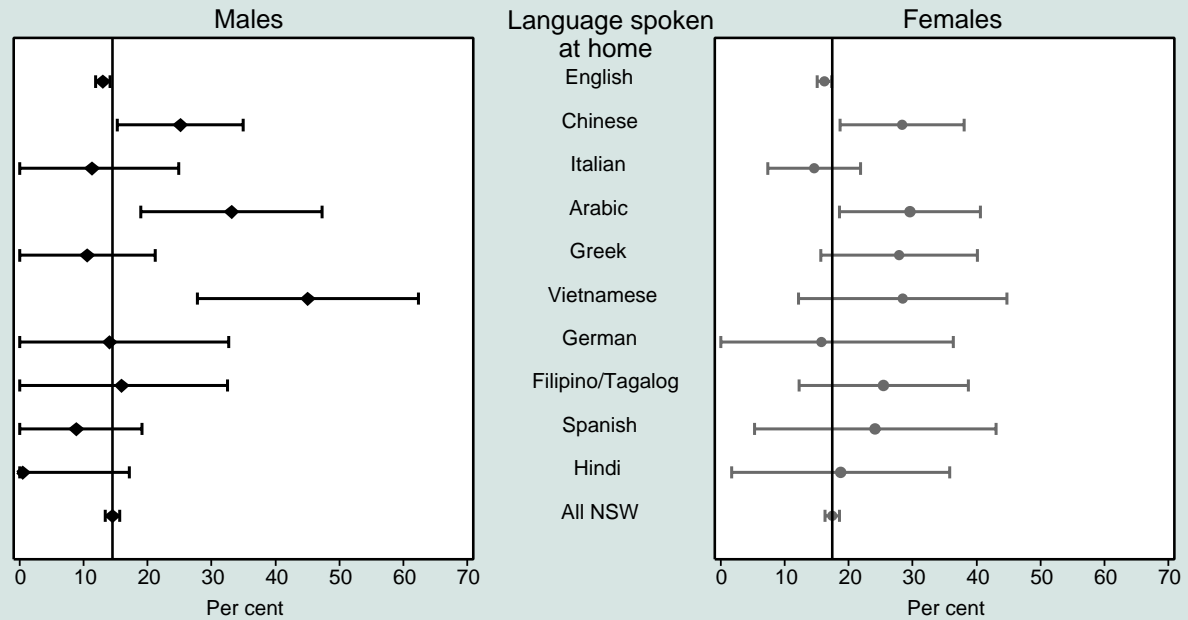

Note: Estimates based on 15,557 respondents with at least one natural tooth ( 0 in $1997 ; 15,557$ in 1998). $36(0.2 \%)$ not stated for toothache in the previous 12 months. 13,870 respondents spoke English at home; 1,669 respondents spoke a language other than English at home.

Source: NSW Health Survey 1998 (HOIST). Epidemiology and Surveillance Branch, NSW Department of Health. 


\section{FIGURE 3}

\section{PSYCHOLOGICAL DISTRESS BY AGE AND INDIGENOUS STATUS}

Psychological distress score of 60 or more by age and indigenous status, persons aged 16 years and over, NSW, 1997 and 1998
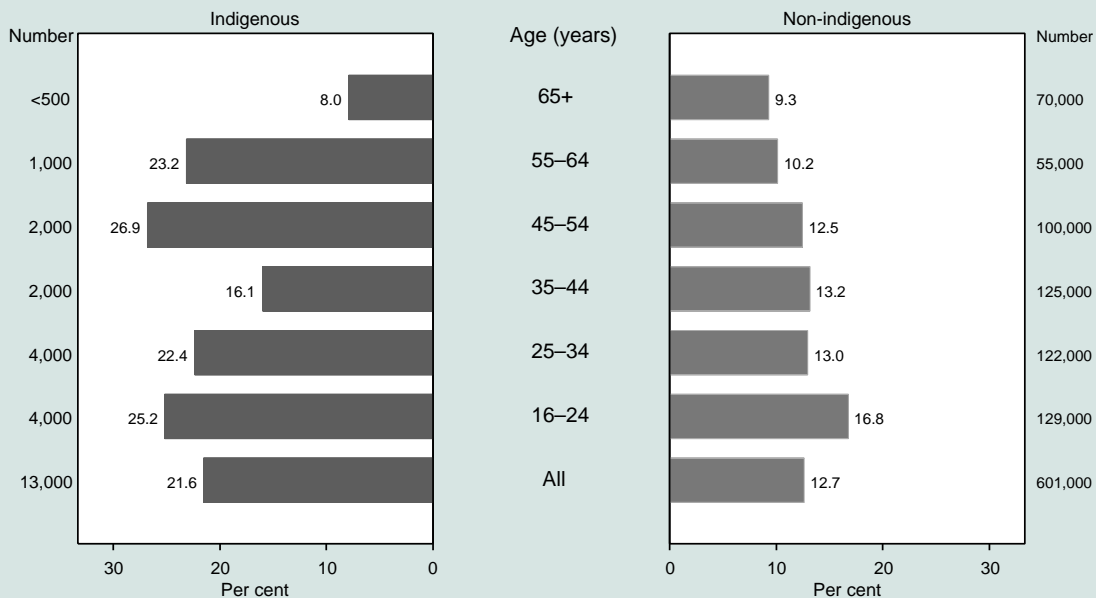

Note: Estimates based on 35,025 respondents (17,531 in 1997; 17,494 in 1998). There were 646 indigenous and 34,360 non-indigenous respondents.

Source: NSW Health Surveys 1997 and 1998 (HOIST). Epidemiology and Surveillance Branch, NSW Department of Health.

\section{FIGURE 4}

DEATHS FROM ISCHAEMIC HEART DISEASE AND HOSPITALISATIONS FOR CORONARY ARTERY BYPASS GRAFTS, BY ACCESSIBILITY-REMOTENESS INDEX FOR AUSTRALIA (ARIA)

Deaths from ischaemic heart disease and hospital separations for coronary artery bypass graft by ARIA, NSW
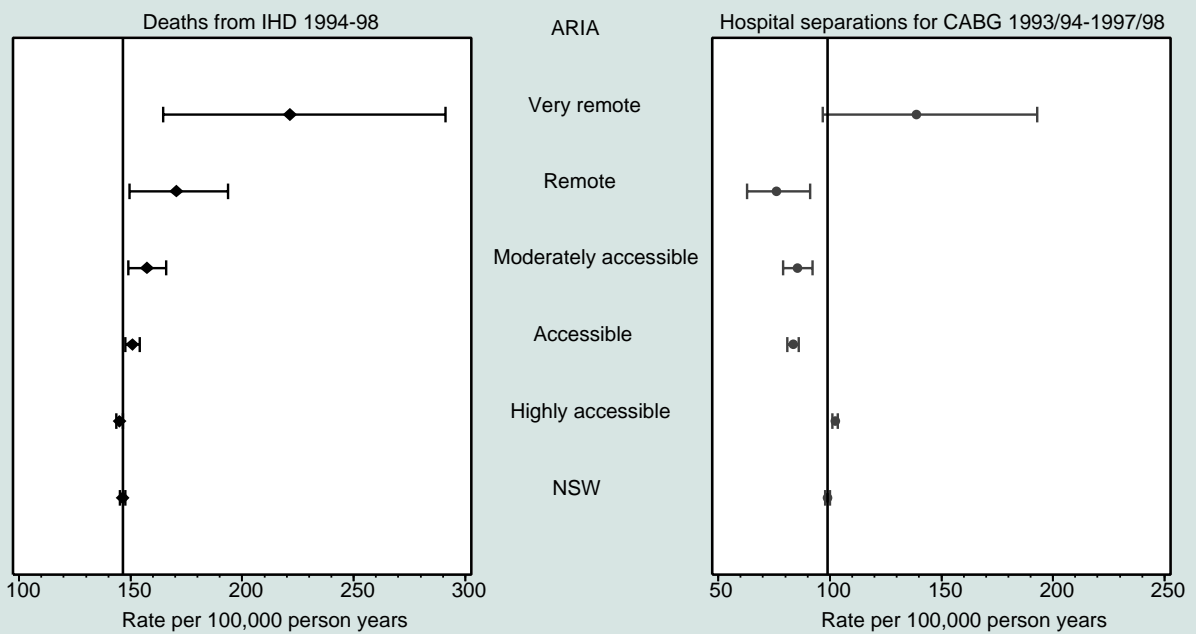

Note: Ischaemic heart disease was classified according to the ICD-9-CM diagnosis codes 410-414. Coronary artery graft was classified according to the ICD-9-CM procedure code 36.1. Statistical local areas were assigned to the Accessibility/Remoteness Index of Australia (ARIA). Rates were age-adjusted using the Australian population as at 30 June 1991. LL/UL95\%Cl of the standardised rate are shown.

Source: ABS mortality data and population estimates (HOIST). Epidemiology and Surveillance Branch, NSW Department of Health. 
as non-indigenous people to rate the care they received in hospital as 'fair' or 'poor' (9.3 per cent).

- In 1997-1998, indigenous people living in rural areas in NSW (162 per 100,000 population) were just over three times more likely to receive haemodialysis than indigenous people living in urban areas (53 per 100,000 population), and five times more likely to receive haemodialysis than non-indigenous people living in rural areas (32 per 100,000 population).

\section{HEALTH INEQUALITIES BY PLACE OF RESIDENCE}

Measurement of health inequalities associated with geographic remoteness has been facilitated by the development of the Accessibility-Remoteness Index for Australia (ARIA). ${ }^{6}$ This is based on road distance travelled from major service centres and provides a measure of service access on a population basis. ARIA scores can be assigned on the basis of postcode of residence. Examples of inequalities demonstrated by analysis by ARIA category include:

- In 1994-1998, death rates from ischaemic heart disease increased progressively with increasing remoteness. By contrast, hospital separation rates for coronary artery bypass graft (CABG) showed a less consistent pattern, with little difference in rates for those living in remote and highly accessible areas, and slightly lower rates for those living in areas with intermediate levels of service access (Figure 4).

- In the 1997 and 1998 NSW Health Surveys, a higher percentage of people living in remote $(60.0$ per cent) and very remote (69.6 per cent) areas of NSW reported one or more alcohol drinking behaviours that are associated with an increased risk to health compared with those living in highly accessible areas (49.0 per cent).

- In the same surveys, a higher percentage of people living in remote (20.8 per cent) and very remote (41.3 per cent) areas of NSW reported having difficulties getting the health care they needed compared with those living in highly accessible areas (8.2 per cent).

\section{HEALTH INEQUALITIES BY SOCIOECONOMIC DISADVANTAGE, LABOUR FORCE CATEGORY AND EDUCATION}

Socioeconomic differentials in health can be measured using data on individuals (for example: level of education, employment status, or income) and relating it to a measure of that individual's health. An alternative approach is to use aggregate socioeconomic characteristics of the populations of defined geographic areas (such as postcodes or local government areas) as a proxy for the socioeconomic status of individuals. ${ }^{3}$ The Socioeconomic Indices for Areas (SEIFA) were developed for this purpose by the

\section{FIGURE 5}

\section{TEENAGE MOTHERS BY INDEX OF RELATIVE SOCIOECONOMIC DISADVANTAGE}

Teenage mothers by socioeconomic disadvantage score for LGAs, NSW 1994 to 1998

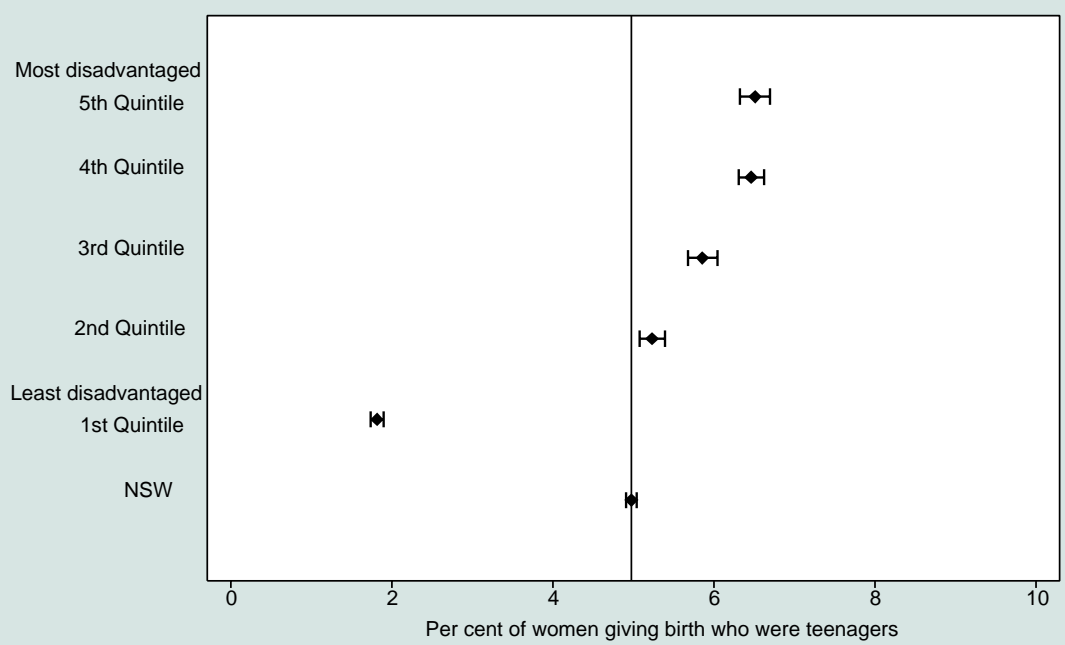

Note: Local Government Areas (LGAs) were classified into quintiles by scores based on the ABS Index of Relative Socioeconomic Disadvantage (IRSD). Lower and upper limits of the 95 per cent confidence interval for the point estimate are shown.

Source: NSW Midwives Data Collection and Census data, and SEIFA index (HOIST). Epidemiology and Surveillance Branch, NSW Department of Health. 


\section{FIGURE 6}

\section{CURRENT SMOKING BY LABOUR FORCE CATEGORY}

Currently smoke daily or occasionally by labour force category and sex, persons aged 16 years and over, NSW 1997 and 1998
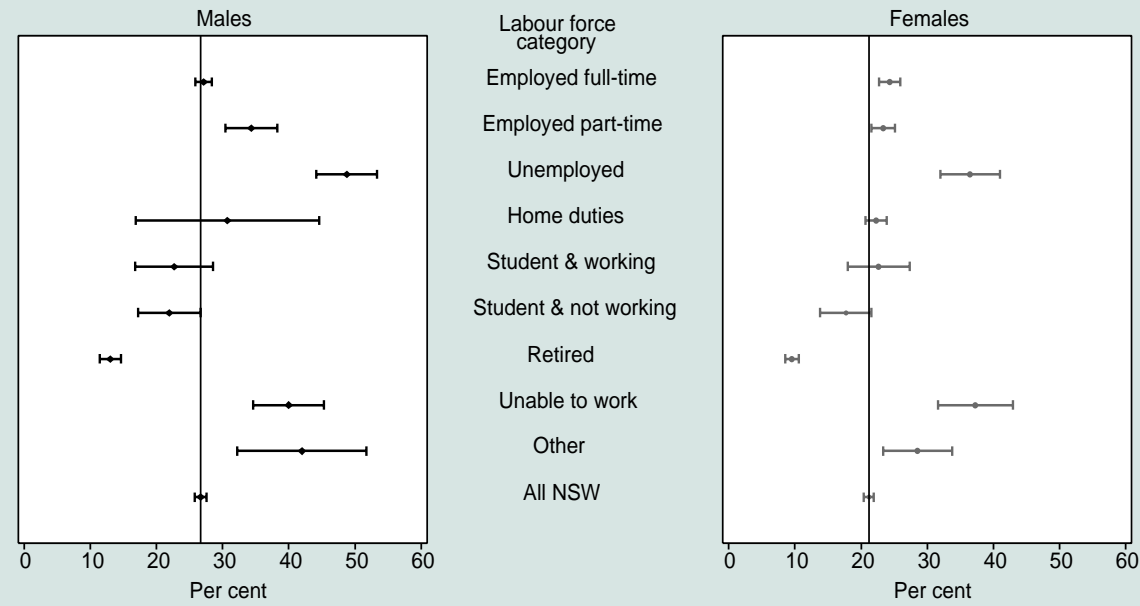

Note: Estimates based on 35,025 respondents (17,531 in 1997; 17,494 in 1998). 6 not stated for current smoking status. Source: NSW Health Surveys 1997 and 1998 (HOIST). Epidemiology and Surveillance Branch, NSW Department of Health.

Australian Bureau of Statistics using census data. ${ }^{7}$ The SEIFA index of relative socioeconomic disadvantage (IRSD) is compiled from 21 different census indicators summarising underlying social and economic variables of disadvantage, such as low income, low level of education, unemployment, recent migration, lack of fluency in English and indigenous status. Socioeconomic differentials demonstrated by analysis of NSW data using both of these approaches include:

- In 1994 to 1998 , the likelihood of giving birth as a teenager was strongly associated with socioeconomic disadvantage. Teenage mothers represented 1.8 per cent of all women giving birth in the least disadvantaged quintile compared with 6.5 per cent of all women giving birth in the most disadvantaged quintile (Figure 5).

- In the 1997 and 1998 NSW Health Surveys, reported rates of current smoking increased with increasing levels of socioeconomic disadvantage. Both male and female respondents who were unable to work, unemployed or employed part-time had much higher reported rates of current smoking than the state average (Figure 6).

- In the same surveys, psychological distress, ${ }^{5}$ was associated with socioeconomic disadvantage. Reported rates of psychological distress were lowest among men and women with university or other tertiary qualifications and highest among respondents who had not completed their high school certificate (Figure 7). It should be noted that the highest level of educational attainment was also strongly associated with age (there is generally a lower level of educational attainment with increasing age).

\section{DISCUSSION}

The reports Health of the people of New South WalesReport of the Chief Health Officer 2000, ${ }^{1}$ and NSW Health Surveys 1997 and 1998, ${ }^{2}$ demonstrate many inequalities in the health of the NSW population, based on sex, ethnicity, indigenous status, area of residence and socioeconomic factors. Whether these differences represent inequities in health relies on an assessment of their fairness and preventability.

Much work is required to improve the measurement of inequalities in health. Issues include the appropriateness of focusing on individual level determinants of health when macrolevel determinants (such as unemployment and income) may have a far greater impact on health and require different policy interventions. ${ }^{9}$ This is particularly important considering evidence that socioeconomic determinants that lead to poor health tend to be concentrated in the same groups in society. ${ }^{10}$

Also, for many conditions, notably non-communicable diseases such as cardiovascular diseases, the relationships between social and economic factors and health are more difficult to understand, and therefore to measure. Here, identifying the role of influences that operate throughout life - the 'lifecourse approach' - may help to tease out differences both between and within socioeconomic groups, which may be different for different conditions. ${ }^{8}$

In future editions of the Report of the Chief Health Officer it is planned to present data on trends in health 


\section{FIGURE 7}

\section{PSYCHOLOGICAL DISTRESS BY LEVEL OF EDUCATION}

Psychological distress score of 60 or more by highest educational attainment and sex, persons aged 16 years and over, NSW 1997 and 1998
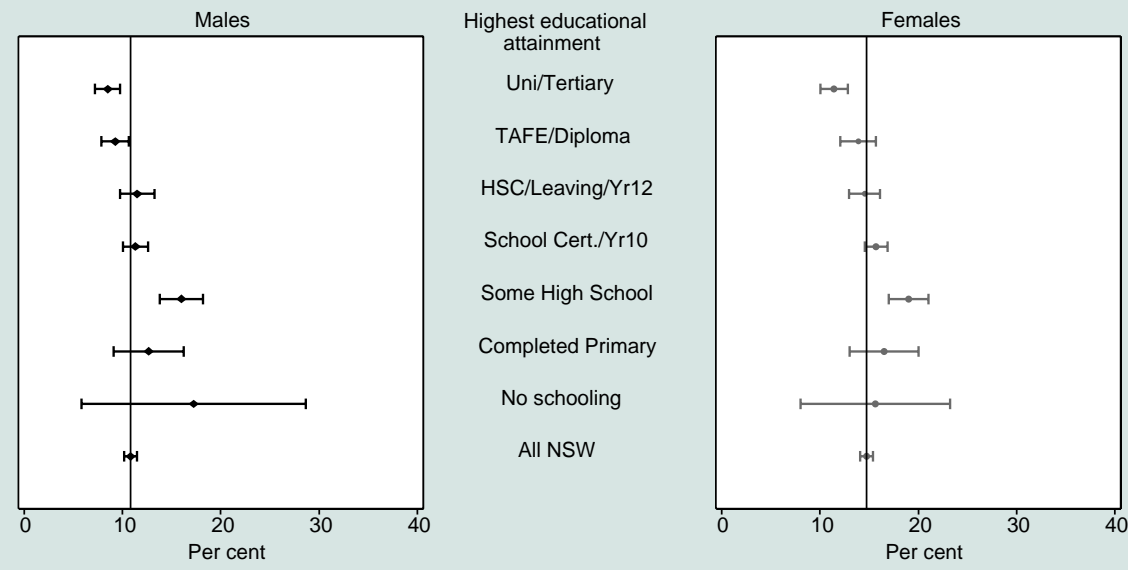

Note: Estimates based on 35,025 respondents (17,531 in 1997; 17,494 in 1998). 290 (0.8\%) not stated for any question in the K10 instrument. Respondents who partially completed primary school are in the no schooling category which had 236 respondents.

Source: NSW Health Surveys 1997 and 1998 (HOIST). Epidemiology and Surveillance Branch, NSW Department of Health.

inequalities. Challenges include choosing indicators for monitoring the size and direction inequalities. A range of such indicators has been described by Mackenbach and Kunst, ${ }^{11}$ and by Gakidou et al. ${ }^{12}$ Selecting which ones to present involves making choices between measures of relative and absolute differences; individual-mean differences and interindividual differences; and simple measures and more sophisticated ones. Ideally, such choices should be informed by eliciting information on community preferences, through mechanisms such as the NSW Health Survey.

\section{ACKNOWLEDGEMENTS}

Past and present staff of the Epidemiology and Surveillance Branch involved in the production of the Health of the people of New South Wales-Report of the Chief Health Officer 2000 and NSW Health Surveys 1997 and 1998 included Deborah Baker, Tim Churches, Devon Indig, Jill Kaldor, Kim Lim, Ru Nguyen, Hanna Noworytko, Tim Owen, Michelle Puech, Lee Taylor and Margaret Williamson. Members of the Health Equity Forum assisted with text about health inequalities for the Report of the Chief Health Officer 2000.

\section{REFERENCES}

1. NSW Department of Health. Health of the people of New South Wales-Report of the Chief Health Officer, 2000. Sydney: NSW Department of Health, 2000.

2. NSW Department of Health. NSW Health Surveys 1997 and 1998. Sydney: NSW Department of Health, 2001; www.health.nsw.gov.au/public-health/nswhs/index.htm.
3. Mathers C. Health Differentials Among Adult Australians aged 25-64 years. Canberra: AIHW Health Monitoring Series, No. 1, 1994.

4. Australian Bureau of Statistics. The Health and Welfare of Australia's Aboriginal and Torres Strait Islander peoples. Canberra: AGPS, 1999. ABS Catalogue no. 4704.0.

5. Kessler R, Mroczec D. Final versions of our Non-Specific Psychological Distress Scale. Ann Arbor, MI: Survey Research Centre of the Institute for Social Research, University of Michigan; Memo dated March 10, 1994.

6. Commonwealth Department of Aged Care and the National Key Centre for Social Applications of Geographical Information Systems (University of Adelaide). AccessibilityRemotness Index of Australia. Canberra: Department of Health and Aged Care, 1999.

7. Australian Bureau of Statistics. Information paper: 1996 Census socioeconomic indices for areas. Canberra: AGPS, 1998. ABS Catalogue no. 29120.

8. Leon D, Walt G, Gilson L. International perspectives on health inequalities and policy. BMJ 2001; 322: 591-4.

9. Davey Smith G, Ebrahim S, Frankel S. How policy informs the evidence. BMJ 2001; 322: 184-5.

10. Vinson T. Unequal in life. The distribution of social disadvantage in Victoria and New South Wales. Melbourne: The Ignatius Centre, 1999.

11. Mackenbach JP, Kunst AE. Measuring the magnitude of socioeconomic inequalities in health: an overview of available measures illustrated with two examples from Europe. Social Science and Medicine 1997; 44: 757-71.

12. Gakidou EE, Murray CJL, Frenk J. Defining and measuring health inequality: an approach based on the distribution of health expectancy. Bulletin of the World Health Organization 2000; 78: 42-54. 